\title{
The adiponectin gene is associated with adiponectin levels but not with characteristics of the insulin resistance syndrome in healthy Caucasians
}

\author{
Vitolds Mackevics ${ }^{1,2}$, Iris M Heid ${ }^{3}$, Stefanie A Wagner ${ }^{1,3}$, Paula Cip ${ }^{1}$, Hildegard \\ Doppelmayr $^{1}$, Aivars Lejnieks ${ }^{2}$, Henning Gohlke ${ }^{3}$, Günther Ladurner ${ }^{4}$, Thomas Illig ${ }^{3}$, \\ Bernhard Iglseder $^{4}$, Florian Kronenberg ${ }^{*, 5}$ and Bernhard Paulweber ${ }^{1}$
}

\footnotetext{
${ }^{1}$ First Department of Internal Medicine, St. Johann Spital, Paracelsus Private Medical University Salzburg, Austria; ${ }^{2}$ Department of Internal Medicine, Faculty of Medicine, Riga Stradins University, Latvia; ${ }^{3}$ GSF-National Research Center for Environment and Health, Institute of Epidemiology, Neuherberg, Germany; ${ }^{4}$ Department of Neurology, Christian-Doppler Klinik, Paracelsus Private Medical University Salzburg, Austria; ${ }^{5}$ Division of Genetic Epidemiology, Department of Medical Genetics, Molecular and Clinical Pharmacology, Innsbruck Medical University, Innsbruck, Austria
}

Low concentrations of adiponectin, the protein product of the APM1 gene, have been reported to be associated with obesity and insulin resistance. However, contrasting results have been described on the genetic variability in APM1 and characteristics of the metabolic syndrome and adiponectin serum concentrations. In the present study, we investigated the association of the two most well-known SNPs of $A P M 1(+45 T>G$ and $+276 G>T)$ and their haplotypes, with serum adiponectin concentrations, metabolic parameters and intima-media thickness of the carotid arteries in 1745 well-phenotyped asymptomatic unrelated Caucasian subjects of the SAPHIR cohort. The common T-allele (88.5\%) of SNP $+45 T>$ G and the common G-allele $(70.5 \%)$ of SNP $+276 \mathrm{G}>\mathrm{T}$ were associated with significantly lower serum adiponectin levels $(P=0.0008$ and $P=0.00005$, respectively). The most frequent haplotype TG (59.0\%) defined by both loci showed a strong association with lower serum adiponectin concentrations $(P=0.000000002)$. A clear effect per copy of the respective haplotype was observed. This association was most pronounced in lean and insulin-sensitive subjects. The two less common haplotypes TT (29.5\%) and GG (11.5\%) were associated with higher serum adiponectin levels in a dose-dependent association.

Interestingly, no significant association between the adiponectin 45-276 haplotypes and the majority of parameters of the metabolic syndrome or intima-media thickness of the carotid arteries was found in our study. In summary, we replicated a strong association of the adiponectin 45-276 genotypes and haplotypes with adiponectin levels in healthy Caucasians. However, we could not confirm an association of this gene locus with metabolic parameters of the insulin resistance syndrome.

European Journal of Human Genetics (2006) 14, 349-356. doi:10.1038/sj.ejhg.5201552; published online 18 January 2006

Keywords: insulin resistance; adiponectin; metabolic syndrome; association study

\footnotetext{
*Correspondence: F Kronenberg, MD, Division of Genetic Epidemiology, Department of Medical Genetics, Molecular and Clinical Pharmacology, Innsbruck Medical University, Schöpfstr. 41, A-6020 Innsbruck, Austria. Tel: + 43512507 3490; Fax: + 43512507 9804;

E-mail: Florian.Kronenberg@i-med.ac.at

Received 27 May 2005; revised 9 November 2005; accepted 10 November 2005; published online 18 January 2006
}

\section{Introduction}

Recent studies point to the adipose tissue as a highly active endocrine organ secreting a range of hormones. There is great interest in clarifying the role of adipose tissue-derived peptides (adipokines) as possible mediators between body fat distribution and insulin sensitivity. 
Adiponectin, the major adipocyte secretory protein, has been shown to improve insulin sensitivity and to possess anti-inflammatory and antiatherosclerotic properties. ${ }^{1-8}$ Hypoadiponectinaemia has been found to be associated with insulin resistance, ${ }^{9,10}$ obesity $^{4,11}$ and other features of the metabolic syndrome, ${ }^{12-14}$ as well as type 2 diabetes ${ }^{15,16}$ and cardiovascular disease. ${ }^{5-8}$ In patients with anorexia nervosa ${ }^{17}$ and chronic renal failure, ${ }^{10,18}$ plasma adiponectin levels are increased. Weight loss and therapy with thiazolidinediones enhance endogenous adiponectin production in humans. ${ }^{19}$

Two common nucleotide changes in the adiponectin gene, SNPs $+45 \mathrm{~T}>\mathrm{G}$ and $+276 \mathrm{G}>\mathrm{T}$, have been shown to be associated with obesity and insulin sensitivity, ${ }^{12,20}$ type 2 diabetes, ${ }^{21-23}$ and coronary artery disease in type 2 diabetic patients, ${ }^{24-26}$ and serum adiponectin levels. ${ }^{12,14,21,22,26-31}$ A haplotype of these two SNPs showed even higher associations with obesity and other components of the metabolic syndrome. ${ }^{12}$ However, the data are inconsistent and many of the described associations above could not be confirmed in other studies. ${ }^{32-36}$ Most studies investigated the association between the APM1 gene and adiponectin levels in diabetic or obese subjects. Only few studies focused on healthy individuals from the general population.

In the present study, we investigated the association of adiponectin 45-276 genotypes and haplotypes with serum adiponectin concentrations and metabolic parameters of the insulin resistance syndrome, in a large ethnically homogeneous and well-phenotyped healthy Austrian population.

\section{Subjects and methods Study participants and clinical investigations}

A total of 1745 subjects were included in the present study. The cohort consisted of 1098 male subjects aged between 39 and 66 years and 647 female subjects aged between 47 and 67 years, who were participants of the 'Salzburg Atherosclerosis Prevention program in subjects at High Individual Risk' (SAPHIR), a prospective study investigating the role of various genetic and metabolic factors for progression of atherosclerotic vascular disease. ${ }^{37}$ All individuals were of Caucasian origin and the population was homogeneous with regard to ethnic background. Subjects with established coronary artery, cerebrovascular or peripheral arterial disease, congestive heart failure, valvular heart disease, chronic alcohol (more than three drinks a day) or drug abuse, morbid obesity $\left(\mathrm{BMI}>40 \mathrm{~kg} / \mathrm{m}^{2}\right)$ and pregnant women were excluded. At baseline all study participants were subjected to a thorough screening program that included assessment of a detailed personal and family history, physical examination, determination of anthropometric indices and measurement of various biochemical parameters and several other procedures, including measurement of the intima-media thickness (IMT) of the carotid arteries by B-mode ultrasound. Subjects were classified as type 2 diabetics if they were on hypoglycemic medications or when their fasting glucose concentrations exceeded $126 \mathrm{mg} / \mathrm{dL}$. Informed consent was obtained from each participant and the study was carried out in accordance with the local ethics committee.

IMT of carotid arteries was measured by high-resolution B-mode ultrasound (HDI 3000 CV from ATL, Munich, Germany) according to the protocol published by the ACAPS' investigators. ${ }^{38}$ All measurements were performed by the same ultrasound operator. Additionally, a B-score describing morphological alterations of the carotid arteries was formed as follows: 0, no alterations; 1 , wall thickness $>1 \mathrm{~mm} ; 2$, plaque $<2 \mathrm{~mm} ; 3$, plaque $2-3 \mathrm{~mm} ; 4$, plaque $>3 \mathrm{~mm} ; 5$, total occlusion of the lumen.

A $24 \mathrm{~h}$ ambulatory blood pressure measurement was performed using the TM 2430 PC monitoring system from BOSO (Bosch + Sohn, Jungingen, Germany). Body composition was determined by body impedance analysis (BIA 2000-S, Data Input, Darmstadt, Germany).

\section{Laboratory measurements}

Venous blood was collected after an overnight fast, and plasma and serum samples were either used immediately for analysis or were stored frozen at $-80^{\circ} \mathrm{C}$. Total serum cholesterol, triglycerides, HDL-cholesterol, LDL-cholesterol, Lp(a), apolipoprotein A-I, apolipoprotein B, urinary albumin/ creatinine ratio, HbA1c and fasting glucose were determined using commercially available assays (Hoffmann-LaRoche $\mathrm{GmbH}$, Vienna, Austria). Fasting insulin was measured using the IMX insulin assay (Abbot, Vienna, Austria). Insulin sensitivity was determined using the short insulin tolerance test (k-ITT). Insulin sensitivity was also estimated by the homeostasis model assessment (HOMA-IR) indices calculated using the formula: fasting blood glucose $(\mathrm{mg} / \mathrm{dl}) \times$ fasting insulin $(\mu \mathrm{U} / \mathrm{ml}) / 405$. Glucose tolerance was evaluated according to the 1985 World Health Organization criteria (oral glucose tolerance test, OGTT). Serum adiponectin levels were measured using the human adiponectin ELISA kit (BioCat GmbH, Heidelberg, Germany). Subjects on lipid-lowering drugs were excluded from statistical analysis of lipids.

\section{Genetic analysis}

Genomic DNA was isolated from white blood cells, using Puregen DNA purification kit (Gentra Systems, MN, USA) and stored at $-20^{\circ} \mathrm{C}$. Genotyping was performed with two different methods in the entire cohort. First, for the SNP $+45 \mathrm{~T}>\mathrm{G}$, a 305-bp DNA fragment containing the polymorphic site was amplified by polymerase chain reaction (PCR) using forward primer 5'- TGT GTG TGT GGG GTC TGT CT- $3^{\prime}$ and reverse primer 5'-TGT GAT GAA AGA GGC CAG AA- $3^{\prime}$. The annealing temperature was $62^{\circ} \mathrm{C}$. After digestion with the restriction enzyme Ava I (incubation 
temperature $37^{\circ} \mathrm{C}$ ), fragments were separated on $2 \%$ agarose gels. For the SNP +276G $>$ T, a 107-bp DNA fragment containing the polymorphic site was amplified by PCR using forward primer $5^{\prime}$-CTA CAC TGA TAT AAA CTA TAT GGA G-3' and reverse primer $5^{\prime}$-CCC CAA ATC ACT TCA GGT TG-3'. The annealing temperature was $55^{\circ} \mathrm{C}$. After digestion with the restriction enzyme Hinf I (incubation temperature $37^{\circ} \mathrm{C}$ ), fragments were separated on $15 \%$ polyacrylamide gels. Bands were visualized by ethidium bromide staining. Second, genotypes of both SNPs were reevaluated using MALDI-TOF-based mass spectrometry of allele specific primer extension products (Mass ArrayTM, Sequenom, San Diego, CA, USA) as described previously. ${ }^{39}$ These two methods showed no major differences. Additionally, $15 \%$ of the samples were genotyped in duplicate for quality control, using MALDI-TOF-based mass spectrometry. Altogether, we found 13 discordant genotypes in 4908 duplicates of recent genotyping projects leading to a discordance rate of $0.26 \%$. Moreover, $2 \%$ of negative and positive controls are added routinely.

\section{Statistical analysis}

Haplotypes were statistically reconstructed using the EMalgorithm implemented in PROC HAPLOTYPE by SAS ${ }^{\circledR}$. Hardy-Weinberg equilibrium was tested using the $\chi^{2}$ test. The association of the genotypes and the haplotypes with adiponectin concentrations was estimated using a general linear regression model. Further, we estimated and tested the change in adiponectin concentrations per copy of one allele/haplotype. Adiponectin concentrations were analysed on a transformed scale $(\log$ (adiponectin +1$))$ in order to obtain a normally distributed variable. A trend per copy of the allele was ascertained by viewing the mean adiponectin concentrations in three groups: subjects with two, one or zero copies of the rare allele or haplotype. All analyses were adjusted for gender, age and BMI. Two-tailed tests were performed with the significance level set at 0.05 .

The association between haplotypes and serum adiponectin concentrations was also evaluated in four clinical subgroups: (1) L-IS - lean and insulin sensitive (BMI $<$ median, $\mathrm{k}-\mathrm{ITT} \geqslant$ median, $n=532$ ), (2) L-IR - lean and insulin resistant (BMI $<$ median, $\mathrm{k}$-ITT $<$ median, $n=335$ ), (3) O-IS - obese and insulin sensitive (BMI $\geqslant$ median, k-ITT $\geqslant$ median, $n=318$ ), (4) O-IR - obese and insulin resistant (BMI $\geqslant$ median, k-ITT $<$ median, $n=560$ ).

In a secondary analysis, the parameters BMI, waist, fat mass, HbA1c, k-ITT values, total cholesterol, HDL and LDL cholesterol, triglycerides, apolipoprotein A-I, apolipoprotein B, Lp(a), systolic and diastolic blood pressure, IMT of the common carotid artery (CCA) and the internal carotid artery (ICA), the albumin-creatinine ratio, HOMAIR, fasting glucose and fasting insulin concentrations as well as the OGTT parameters (glucose and insulin concentrations $1 / 2,1$ and $2 \mathrm{~h}$ after OGTT) were tested using a general linear regression model with regard to their association with the APM1 +45 and +276 loci. Adiponectin concentrations, all insulin concentrations, triglycerides, $\operatorname{Lp}(\mathrm{a})$, the albumin-creatinine ratio and the HOMA-IR index were logarithmically transformed in order to more closely fulfill the requirements of a normal distribution.

\section{Results}

Clinical characteristics of 1098 male and 647 female participants of the SAPHIR study are summarized in Table 1. The proportion of subjects with type 2 diabetes was $3.8 \%$ among males and $2.3 \%$ among females.

In Table 2, frequencies of genotypes and haplotypes are summarized. The rare allele $\mathrm{G}$ of the APM1 +45 locus showed a minor allele frequency (MAF) of $11.5 \%$. The rare allele $\mathrm{T}$ of the locus +276 had an MAF of $29.5 \%$. Lewontin's D' of these two loci was unity; the correlation between the alleles was 0.23 with the minor $G$ allele of the +45 locus being correlated with the major $G$ allele of the +276 locus. The TG haplotype was the most common haplotype, with a frequency of 59\%, among the chromosomes. The other two haplotypes in this sample were the TT $(29.5 \%)$ and the GG (11.5\%).

The table further states the mean (95\% confidence intervals) serum adiponectin concentrations per genotype/haplotype. These rescaled means were derived via a general linear model adjusting for age and sex performed on $\log ($ adiponectin +1$)$. The $P$-values testing for association of a single nucleotide polymorphism (SNP) locus or for association of a haplotype with adiponectin concentrations without and with assuming a trend per copy of the allele/haplotype are given. It can be seen that there is a highly significant association of both the +45 and the +276 locus $(P=0.002$ and 0.0002 without assuming a

Table 1 Clinical characteristics of the SAPHIR study population

\begin{tabular}{lcc}
\hline & Males $(n=1098)$ & Females $(n=647)$ \\
\hline Age (years) & $49.2 \pm 5.5$ & $56.2 \pm 4.3$ \\
BMl (kg/m $\left.\mathrm{m}^{2}\right)$ & $26.92 \pm 3.72$ & $26.59 \pm 4.74$ \\
Smokers (\%) & 22.9 & 13.3 \\
Diabetes (\%) & 3.8 & 2.3 \\
HbA1c $(\%)$ & $5.60 \pm 0.59$ & $5.66 \pm 0.46$ \\
Fasting glucose $(\mathrm{mg} / \mathrm{dl})$ & $94.4 \pm 18.3$ & $92.1 \pm 17.1$ \\
Fasting insulin $(\mu \mathrm{U} / \mathrm{ml})$ & $7.57 \pm 5.56$ & $7.23 \pm 4.27$ \\
Total cholesterol $(\mathrm{mg} / \mathrm{dl})$ & $226 \pm 40$ & $234 \pm 41$ \\
LDL cholesterol $(\mathrm{mg} / \mathrm{dl})$ & $146 \pm 36$ & $145 \pm 38$ \\
HDL cholesterol $(\mathrm{mg} / \mathrm{dl})$ & $55.2 \pm 13.4$ & $67.1 \pm 16.4$ \\
Triglycerides $(\mathrm{mg} / \mathrm{dl})$ & $137 \pm 100$ & $106 \pm 53$ \\
SBP $(\mathrm{mmHg})$ & $135 \pm 13$ & $131 \pm 13$ \\
DBP $(\mathrm{mmHg})$ & $82 \pm 8$ & $81 \pm 8$ \\
\hline The values are & &
\end{tabular}

The values are given as mean \pm standard deviation. For the dichotomously coded smokers and diabetics, percentages are given. 
Table 2 Association of the $+45 \mathrm{~T}>\mathrm{G}$ and $+276 \mathrm{G}>\mathrm{T}$ polymorphisms with adiponectin concentrations

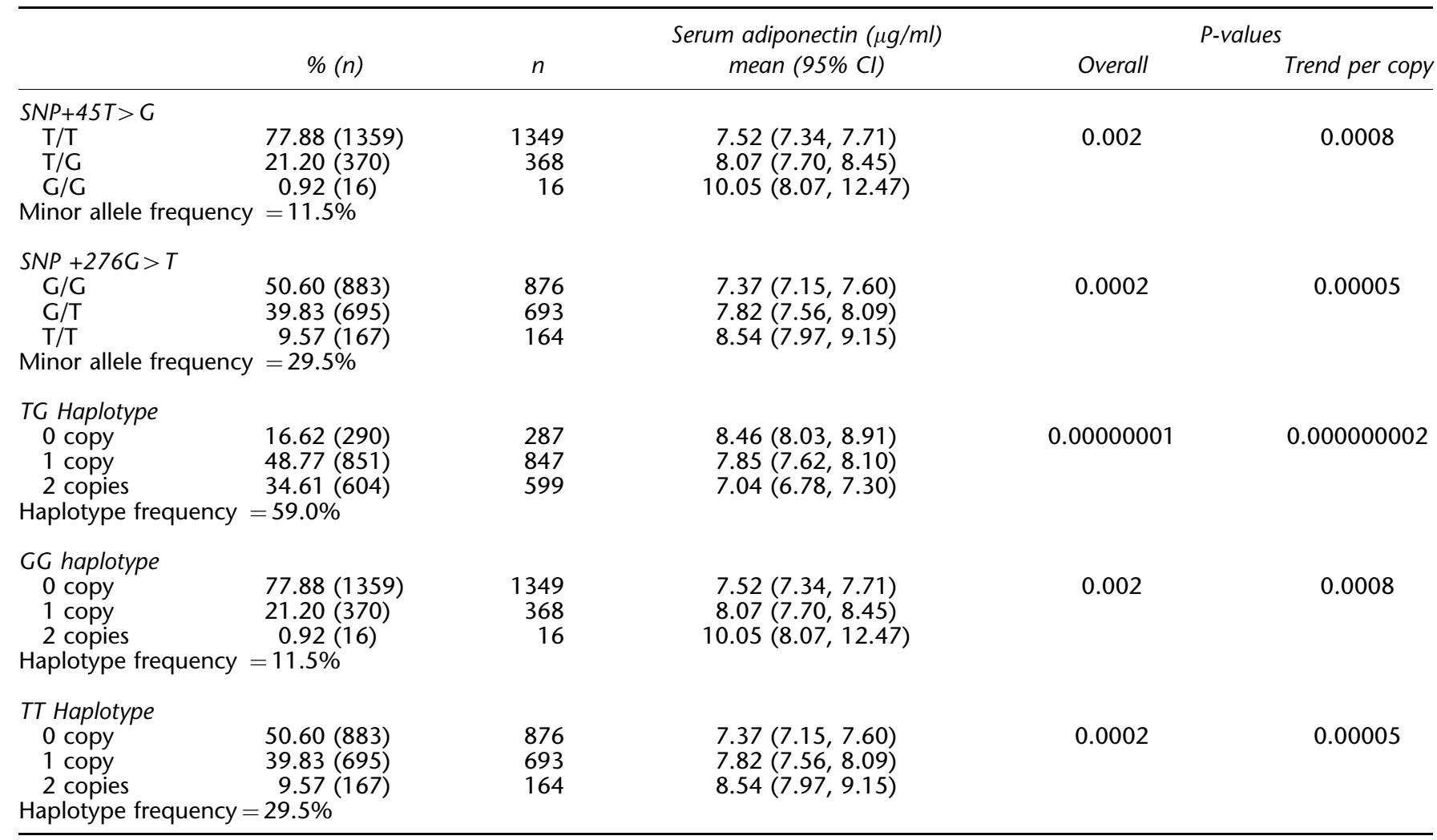

The analysis was performed on the normally distributed transformed variable $x=\log ($ adiponectin +1 ) and adjusted for age and gender, using a general linear model. Values given are genotype/haplotype frequencies, including the number of subjects, re-transformed age-and sex-adjusted mean adiponectin levels including $95 \%$ confidence intervals (ie $\exp (\operatorname{mean}(\mathrm{x}))-1)$, and - for each SNP and each haplotype $-P$-values testing for overall association and a $P$-value testing for a trend per copy of the allele/haplotype.

trend per copy) with adiponectin levels. A clear multiplicative trend of increasing adiponectin levels per copy of the rare allele can be observed $(7.52,8.07,10.05$ for the $\mathrm{T} / \mathrm{T}$, the T/G and the G/G genotype in the +45 locus, $P$-values for testing for trend $=0.0008$, and $7.37,7.82,8.54$ for the G/G, G/T and the T/T genotype in the +276 locus, $P$-value for testing for trend $=0.00005)$. The highest association was observed when analyzing both SNPs together; the haplotype with the major allele in both loci (TG) was most highly associated with decreased adiponectin concentrations showing a clear trend per copy $(8.46,7.85$ and 7.04 for zero, one and two copies of TG, $P=0.00000001$ without trend and $P=0.000000002$ with trend). The other two haplotypes (TT and GG) with one major and one minor allele were associated with increased adiponectin levels. The haplotype with both minor alleles (GT) did not occur in this sample.

All analyses were adjusted for age and sex. Adjustment for age did neither change mean adiponectin levels nor the gene effect. Adjustment for sex did affect the mean values as adiponectin levels differ between male and female subjects. However, the gene effect did not depend upon sex, and thus the genotype/haplotype associations were not stated separately for male and female subjects. Adjustment for BMI did slightly reduce the strength of the association and the difference of the mean adiponectin levels between the genotype/haplotype groups, but the effect of adjusting for BMI was relatively small compared to the strong gene effect of adiponectin ( $P$-value reaching 0.000000002 with a trend per copy).

We also evaluated association of the TG haplotype with serum adiponectin levels in four subgroups: (1) L-IS, lean insulin-sensitive subjects, (2) L-IR, lean insulin-resistant, (3) O-IS, obese insulin-sensitive, (4) O-IR, obese insulinresistant individuals (Table 3 ). The association of the TG haplotype with decreasing adiponectin concentrations was most pronounced in the lean insulin-sensitive subjects $(P \leqslant 0.0001$ assuming a trend per copy). The association was weaker in the lean insulin-resistant subjects $(P=0.005)$, and it was the least obvious in the obese insulin-sensitive subjects $(P=0.04)$ and in the obese insulin-resistant subjects $(P=0.03)$. It can clearly be seen that the differences in adiponectin levels between the homozygous common haplotype and the homozygous rare haplotype, 
Table 3 Association of the 45-276 haplotype and adiponectin concentrations in BMI + k-ITT categorized subgroups

\begin{tabular}{|c|c|c|c|c|}
\hline \multirow[b]{2}{*}{ Groups } & \multicolumn{2}{|c|}{ Lean } & \multicolumn{2}{|c|}{ Obese } \\
\hline & $\begin{array}{c}\text { Insulin sensitive } \\
\quad N=532\end{array}$ & $\begin{array}{c}\text { Insulin resistant } \\
\quad N=335\end{array}$ & $\begin{array}{c}\text { Insulin sensitive } \\
\quad N=318\end{array}$ & $\begin{array}{c}\text { Insulin resistant } \\
N=560\end{array}$ \\
\hline \multicolumn{5}{|c|}{ Mean values and $95 \%$ confidence interval ${ }^{a}$} \\
\hline $\mathrm{BMI}\left(\mathrm{kg} / \mathrm{m}^{2}\right)$ & $23.7(23.4,23.9)$ & $23.7(23.4,24.0)$ & $29.1(28.7,29.4)$ & $30.3(30.1,30.6)$ \\
\hline $\mathrm{k}-\mathrm{ITT}(\% / \mathrm{min})$ & $5.30(5.24,5.37)$ & $3.31(3.23,3.40)$ & $5.05(4.96,5.13)$ & $2.97(2.91,3.04)$ \\
\hline Adiponectin $(\mu \mathrm{g} / \mathrm{ml})$ & $8.59(8.28,8.92)$ & $8.15(7.77,8.54)$ & $7.66(7.29,8.04)$ & $6.60(6.36,6.86)$ \\
\hline \multicolumn{5}{|l|}{ Haplotype frequency: } \\
\hline TG/TG n (\%) & $183(34.4)$ & $125(37.3)$ & $108(34.0)$ & $188(33.6)$ \\
\hline $\mathrm{TG} / \mathrm{X} \mathrm{n}(\%)$ & $258(48.5)$ & $153(45.7)$ & $153(48.1)$ & $287(51.2)$ \\
\hline $\mathrm{X} / \mathrm{X} \mathrm{n}(\%)$ & $91(17.1)$ & $57(17.0)$ & $57(17.9)$ & $85(15.2)$ \\
\hline \multicolumn{5}{|c|}{ Mean serum adiponectin ${ }^{\mathrm{a}}$ by TG haplotype group } \\
\hline $\mathrm{TG} / \mathrm{TG}$ & $8.01(7.51,8.54)$ & $7.89(7.23,8.59)$ & $7.50(6.90,8.13)$ & $6.61(6.20,7.04)$ \\
\hline $\mathrm{TG} / \mathrm{X}$ & $9.45(8.95,9.98)$ & $8.92(8.26,9.65)$ & $8.41(7.85,9.00)$ & $7.00(6.65,7.37)$ \\
\hline$X / X$ & $10.33(9.46,11.28)$ & $9.64(8.50,10.92)$ & $8.51(7.62,9.50)$ & $7.44(6.79,8.15)$ \\
\hline P-value without trend & $P \leqslant 0.0001$ & $P=0.02$ & $P=0.06$ & $P=0.10$ \\
\hline P-value with trend per TG copy & $P \leqslant 0.0001$ & $P=0.005$ & $P=0.04$ & $P=0.03$ \\
\hline
\end{tabular}

L-IS - lean insulin sensitive. (BMI $<$ median, $k-I T T \geqslant$ median); L-IR - lean insulin resistant (BMI $<$ median, $k-I T T<$ median); O-IS - obese insulin-sensitive $(\mathrm{BMI} \geqslant$ median, $\mathrm{k}-\mathrm{ITT} \geqslant$ median); $\mathrm{O}-\mathrm{IR}$ - obese insulin-resistant (BMI $\geqslant$ median, $\mathrm{k}-\mathrm{ITT}<$ median). All median are agegroup- and sex-specific.

$\mathrm{X}$ - denotes any non-TG haplotype.

${ }^{a}$ Means are adjusted for 5-year-age groups and sex, using a general linear model.

in each of these subgroups became smaller with insulin resistance and obesity (ranging from $2.3 \mu \mathrm{g} / \mathrm{ml}$ in the lean insulin-sensitive individuals to about $0.8 \mu \mathrm{g} / \mathrm{ml}$ in the obese insulin-resistant individuals). In the full group using the general linear model as before and including an interaction of the TG haplotype effect with obesity (BMI $>$ median) or insulin resistance (k-ITT $<$ median), the interaction term was highly significant with $P<0.0001$. This further underscores the effect modification of the genotype impact on adiponectin concentrations by obesity and insulin resistance.

In a secondary analysis, a series of biochemical and clinical parameters (BMI, waist, fat mass, HbA1c, k-ITT values, total, $\mathrm{HDL}$ and $\mathrm{LDL}$ cholesterol, triglycerides, apolipoprotein A-I, apolipoprotein B, Lp(a), systolic and diastolic blood pressure, IMT-CCA, IMT-ICA, the albumincreatinine ratio, HOMA-IR, fasting glucose and fasting insulin concentrations as well as glucose and insulin concentrations $1 / 2,1$ and $2 \mathrm{~h}$ after OGTT) were analyzed with respect to their association with the TG haplotype (Table 4). Only apolipoprotein A-I and the albumincreatinine ratio showed a $P$-value of 0.03 , which cannot be deemed significant in the light of the multiple comparisons made (27 parameters tested). IMT of carotid arteries measured by B-mode ultrasound did not show significant differences or even trends in individuals with various 45-276 haplotypes. There was a tendency towards increased glucose concentrations after oral glucose tolerance test (OGTT) with each copy of the TG haplotype (Table 4). Furthermore, the detectable effect size with $80 \%$ power for the given sample size is stated in Table 4.

\section{Discussion}

Several susceptibility loci for type 2 diabetes have been identified by genome wide linkage scans. ${ }^{40}$ Among these regions, 3q27 appears to be of special interest, because it harbours the APM1-gene-encoding adiponectin, which is known to modulate insulin sensitivity and glucose homeostasis. A series of SNPs in the adiponectin gene have been reported. Two common SNPs, a silent $\mathrm{T}>\mathrm{G}$ substitution in exon $2(+45 \mathrm{~T}>\mathrm{G})$ and a $\mathrm{G}>\mathrm{T}$ substitution in intron 2 $(+276 \mathrm{G}>\mathrm{T})$, have been the subject of interest in several investigations. $^{12,14,20-22,26-31,33,41}$ From these studies, it was concluded that both SNPs are related to obesity, insulin resistance and type 2 diabetes. Even stronger associations with traits of the insulin resistance syndrome were reported when the two SNPs were considered together as haplotypes. The haplotype TG defined by these two SNPs has been found to be associated with higher plasma insulin and lower adiponectin levels in healthy individuals. ${ }^{12}$ How these two SNPs and the respective haplotypes are involved in the observed association has to be determined. It is conceivable that the two SNPs are in strong linkage disequilibrium with other SNPs that might by themselves explain the observed association with adiponectin concentrations. It is interesting to note that the TG haplotype is in almost complete linkage disequilibrium with an insertion/deletion polymorphism in the $3^{\prime}$ UTR (position 2019). ${ }^{12}$ As the $3^{\prime}$ UTR region plays a 
Table 4 Association of clinical and metabolic characteristics of the SAPHIR study population with adiponectin 45-276 haplotype TG (X denoting any other haplotypes): stated are mean values and standard deviations

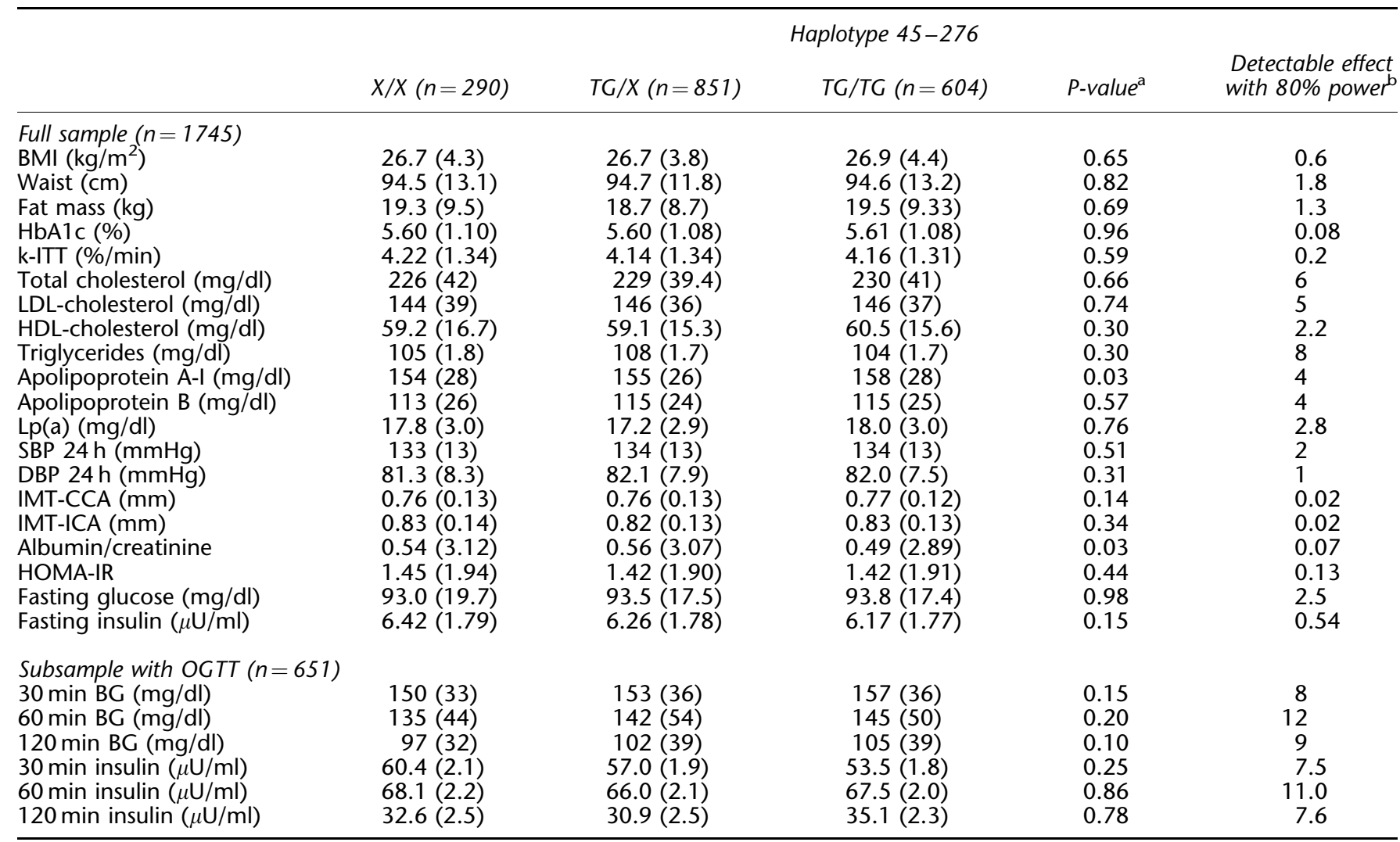

To comply with normality assumption, the following variables were log-transformed: all insulin measures, HbA1c, TG, HOMA-IR, albumin/creatinine and $\operatorname{Lp}(\mathrm{a})$; for these, the geometric mean and geometric standard deviation are given to report on the original scale.

IMT-CCA = intima media thickness of the common carotid arteries; IMT-ICA =intima media thickness of the internal carotid arteries; $\mathrm{HOMA}=$ homeostasis model assessment for insulin resistance; $\mathrm{k}-\mathrm{ITT}=$ short insulin tolerance test; $\mathrm{BG}=$ blood glucose concentration; OGTT $=$ oral glucose tolerance test.

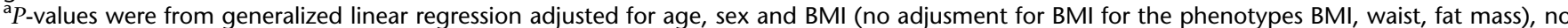
adjustment for multiple testing.

${ }^{\mathrm{b}}$ For the log-transformed variables, the effect size $\delta$ is computed on the log-scale and retransformed by reporting the average between exp $(\mathrm{mean}+\delta)$ $\exp ($ mean $)$ and $\exp ($ mean $-\delta)$ - $\exp ($ mean $)$, where 'mean' is the mean of the variable on the log-scale.

key role in the control of gene expression by binding proteins that regulate mRNA processing, translation or degradation, it could have a strong influence on the investigated protein and related diseases. ${ }^{42}$

Haplotype data from 1745 individuals were available for analysis in our study. The most important finding of our study is the highly demanded replication of an association between the adiponectin $+45 \mathrm{~T}$ allele and the $+276 \mathrm{G}$ allele as well as the 45-276 haplotype TG with significantly lower serum adiponectin levels. It is the highest association for adiponectin levels ever reported, and it is gene-dose-dependent. Two important points have to be discussed in this context.

Genetics of adiponectin levels in a healthy population We consider it as a major strength of the SAPHIR study, that it consists of quite a healthy working population and excluded by definition individuals such as those with established atherosclerotic events, congestive heart failure or morbid obesity. Therefore, associations observed between the APM1 gene and adiponectin levels as observed in our study can be considered not simply as a companion of a disturbed metabolism of such patients but can already be observed long before the development of such diseases. In order to study in more detail, the association between the TG haplotype and insulin sensitivity, we analysed the impact of the TG haplotype in four subgroups, according to BMI as well as k-ITT values as a measure of insulin sensitivity. The association between the 45-276 haplotype and serum adiponectin levels was most pronounced in the subgroup of lean insulin-sensitive individuals $(P<0.0001)$. This is supported by a significant interaction of the TG haplotype with obesity and insulin resistance. One possible explanation for this finding might be that the effect of the 
adiponectin gene locus on serum adiponectin levels is partially over-ruled by environmental factors such as dietinduced obesity. The mentioned subgroup analysis might even explain why we found the strongest associations between genetic variability of these two loci and adiponectin levels, ever reported and which might be strongly related to the generally quite healthy condition of the entire group investigated. In this context, it appears of interest that Filippi et $a l^{32}$ recently reported an association between the adiponectin $276 \mathrm{G}>\mathrm{T}$ genotype and insulin resistance determined by HOMA-IR in a study of 253 nondiabetic subjects. This association was only found in the group of participants with a BMI below the median of $26.2 \mathrm{~kg} / \mathrm{m}^{2}$ with a relative risk of 9.7 and a wide $95 \%$ confidence interval (1.32-87.7).

\section{APM1 and aspects of the metabolic syndrome}

Data from Menzaghi et $a l^{12}$ in 413 nondiabetic individuals suggested that the TG haplotype is strongly associated with insulin resistance parameters. Although we observed a strong association between genetic variations in the APM1 gene and adiponectin levels, we could not detect an association between the 45-276 TG haplotype and numerous metabolic parameters related to insulin resistance. Several circumstances might have contributed to this observation. First, there might be several genes, which have an influence on adiponectin levels and the APM1 gene is only one gene regulating adiponectin concentrations. Second, metabolic parameters of the insulin resistance syndrome are not only determined by serum adiponectin levels, but are influenced by other multiple genetic and environmental factors. Therefore, genetic variations within one gene, explaining a particular amount of an intermediate phenotype such as adiponectin levels, will only be responsible for a very small fraction of end-phenotypes of the metabolic syndrome. To detect this small fraction will need an enormous number of study participants. Power calculations, however, revealed that our study would have been able to detect relatively small and clinically relevant differences between the various haplotypes as shown in Table 4. Third, as mentioned above, the SAPHIR Study consists of a relatively healthy and young study population (average age of 49 and 56 years for men and women, respectively) with a frequency of diabetes mellitus of $3.8 \%$ and $2.3 \%$ for male and female subjects, respectively. Therefore, less marked differences in the variability of the metabolic parameters could be an explanation. We have to bear in mind, that probably a large segment of this population, quite young and healthy at the time of recruitment, has still the potential to develop characteristic features of the metabolic syndrome. A recently published study by Daimon et l $^{16}$ identified a lower serum adiponectin level as an independent risk factor for progression to type 2 diabetes. During a 5-year follow-up period, subjects with serum adiponectin concentrations in the lowest tertile $(<6.46 \mu \mathrm{g} / \mathrm{ml})$ had an almost 10-fold higher risk for development of type 2 diabetes than that for subjects in the upper tertile $(>10.56 \mu \mathrm{g} / \mathrm{ml})$. Results from the ARIC study point still in the same direction but with a less pronounced association. ${ }^{15}$ In light of those studies a difference of $1.42 \mu \mathrm{g} / \mathrm{ml}$ between TG homozygotes and non-TG haplotype carriers as observed in our study might be considered as clinically significant and results in increased risk for diabetes development. A long-term follow-up of our study population is needed to prove this assumption.

In summary, our data show a very strong association of the adiponectin 45-276 haplotypes with adiponectin levels in healthy Caucasians but no association with metabolic parameters of the insulin resistance syndrome.

\section{Acknowledgements}

The SAPHIR study is supported by a Grant to B Paulweber from the Kamillo Eisner-Stiftung, Switzerland. Parts of this work were supported by the German Ministry of Education and Research (BMBF)/National Genome Research Network (NGFN), the Deutsche Forschungsgemeinschaft (SFB 386 and Wi621/12-1) as well as the 'Genomics of Lipid-associated Disorders - GOLD' of the 'Austrian Genome Research Programme GEN-AU'. Vitolds Mackevics received financial support from the 'Verein zur Förderung von Forschung und Lehre an der Landesklinik für Innere Medizin I'.

\section{References}

1 Rabin KR, Kamari Y, Avni I, Grossman E, Sharabi Y: Adiponectin: linking the metabolic syndrome to its cardiovascular consequences. Expert Rev Cardiovasc Ther 2005; 3: 465-471.

2 Berg AH, Scherer PE: Adipose tissue, inflammation, and cardiovascular disease. Circ Res 2005; 96: 939-949.

3 Axelsson J, Heimburger O, Lindholm B, Stenvinkel P: Adipose tissue and its relation to inflammation: the role of adipokines. J Ren Nutr 2005; 15: 131-136.

4 Valle M, Martos R, Gascon F, Canete R, Zafra MA, Morales R: Lowgrade systemic inflammation, hypoadiponectinemia and a high concentration of leptin are present in very young obese children, and correlate with metabolic syndrome. Diabetes Metab 2005; 31: $55-62$.

5 Maahs DM, Ogden LG, Kinney GL et al: Low plasma adiponectin levels predict progression of coronary artery calcification. Circulation 2005; 111: 747-753.

6 Chen MP, Tsai JC, Chung FM et al: Hypoadiponectinemia is associated with ischemic cerebrovascular disease. Arterioscler Thromb Vasc Biol 2005; 25: 821-826.

7 Kumada M, Kihara S, Sumitsuji S et al: Association of hypoadiponectinemia with coronary artery disease in men. Arterioscler Thromb Vasc Biol 2003; 23: 85-89.

8 Pischon T, Girman CJ, Hotamisligil GS, Rifai N, Hu FB, Rimm EB: Plasma adiponectin levels and risk of myocardial infarction in men. JAMA 2004; 291: 1730-1737.

9 Weyer C, Funahashi T, Tanaka S et al: Hypoadiponectinemia in obesity and type 2 diabetes: close association with insulin resistance and hyperinsulinemia. J Clin Endocrinol Metab 2001; 86: $1930-1935$.

10 Zoccali C, Mallamaci F, Tripepi G et al: Adiponectin, metabolic risk factors, and cardiovascular events among patients with endstage renal disease. J Am Soc Nephrol 2002; 13: 134-141. 
11 Havel PJ: Control of energy homeostasis and insulin action by adipocyte hormones: leptin, acylation stimulating protein, and adiponectin. Curr Opin Lipidol 2002; 13: 51-59.

12 Menzaghi C, Ercolino T, Di PR et al: A haplotype at the adiponectin locus is associated with obesity and other features of the insulin resistance syndrome. Diabetes 2002; 51: 2306-2312.

13 Kazumi T, Kawaguchi A, Sakai K, Hirano T, Yoshino G: Young men with high-normal blood pressure have lower serum adiponectin, smaller LDL size, and higher elevated heart rate than those with optimal blood pressure. Diabetes Care 2002; 25: 971-976.

14 Gonzalez-Sanchez JL, Zabena CA, Martinez-Larrad MT et al: An SNP in the adiponectin gene is associated with decreased serum adiponectin levels and risk for impaired glucose tolerance. Obes Res 2005; 13: 807-812.

15 Duncan BB, Schmidt MI, Pankow JS et al: Adiponectin and the development of type 2 diabetes: the atherosclerosis risk in communities study. Diabetes 2004; 53: 2473-2478.

16 Daimon M, Oizumi T, Saitoh T et al: Decreased serum levels of adiponectin are a risk factor for the progression to type 2 diabetes in the Japanese Population: the Funagata study. Diabetes Care 2003; 26: 2015-2020.

17 Delporte ML, Brichard SM, Hermans MP, Beguin C, Lambert M: Hyperadiponectinaemia in anorexia nervosa. Clin Endocrinol (Oxford) 2003; 58: 22-29.

18 Becker B, Kronenberg F, Kielstein JT et al: Renal insulin resistance syndrome, adiponectin and cardiovascular events in patients with kidney disease: the Mild and Moderate Kidney Disease Study. J Am Soc Nephrol 2005; 16: 1091-1098.

19 Maeda N, Takahashi M, Funahashi T et al: PPARgamma ligands increase expression and plasma concentrations of adiponectin, an adipose-derived protein. Diabetes 2001; 50: 2094-2099.

20 Stumvoll M, Tschritter O, Fritsche A et al: Association of the T-G polymorphism in adiponectin (exon 2) with obesity and insulin sensitivity: interaction with family history of type 2 diabetes. Diabetes 2002; 51: 37-41.

21 Hara K, Boutin P, Mori Y et al: Genetic variation in the gene encoding adiponectin is associated with an increased risk of type 2 diabetes in the Japanese population. Diabetes 2002; 51: $536-540$.

22 Fumeron F, Aubert R, Siddiq A et al: Adiponectin gene polymorphisms and adiponectin levels are independently associated with the development of hyperglycemia during a 3-year period: the epidemiologic data on the insulin resistance syndrome prospective study. Diabetes 2004; 53: 1150-1157.

23 Zacharova J, Chiasson JL, Laakso M: The common polymorphisms (single nucleotide polymorphism [SNP] +45 and SNP +276) of the adiponectin gene predict the conversion from impaired glucose tolerance to type 2 diabetes: the STOP-NIDDM trial. Diabetes 2005; 54: 893-899.

24 Bacci S, Menzaghi C, Ercolino T et al: The +276 G/T single nucleotide polymorphism of the adiponectin gene is associated with coronary artery disease in type 2 diabetic patients. Diabetes Care 2004; 27: 2015-2020.

25 Lacquemant C, Froguel P, Lobbens S, Izzo P, Dina C, Ruiz J: The adiponectin gene $\mathrm{SNP}+45$ is associated with coronary artery disease in Type 2 (non-insulin-dependent) diabetes mellitus. Diabet Med 2004; 21: 776-781.

26 Qi L, Li T, Rimm E et al: The +276 polymorphism of the APM1 gene, plasma adiponectin concentration, and cardiovascular risk in diabetic men. Diabetes 2005; 54: 1607-1610.

27 Menzaghi C, Ercolino T, Salvemini L et al: Multigenic control of serum adiponectin levels: evidence for a role of the APM1 gene and a locus on 14q13. Physiol Genomics 2004; 19: 170-174.

28 Vasseur F, Helbecque N, Dina C et al: Single-nucleotide polymorphism haplotypes in the both proximal promoter and exon 3 of the APM1 gene modulate adipocyte-secreted adiponectin hormone levels and contribute to the genetic risk for type 2 diabetes in French Caucasians. Hum Mol Genet 2002; 11: 2607-2614.

29 Berthier MT, Houde A, Cote $\mathrm{M}$ et al: Impact of adiponectin gene polymorphisms on plasma lipoprotein and adiponectin concentrations of viscerally obese men. J Lipid Res 2005; 46: 237-244.

30 Gibson F, Froguel P: Genetics of the APM1 locus and its contribution to type 2 diabetes susceptibility in French Caucasians. Diabetes 2004; 53: 2977-2983.

31 Pollin TI, Tanner K, O'Connell JR et al: Linkage of plasma adiponectin levels to 3q27 explained by association with variation in the APM1 gene. Diabetes 2005; 54: 268-274.

32 Filippi E, Sentinelli F, Trischitta V et al: Association of the human adiponectin gene and insulin resistance. Eur J Hum Genet 2004; 12: $199-205$.

33 Lee YY, Lee NS, Cho YM et al: Genetic association study of adiponectin polymorphisms with risk of Type 2 diabetes mellitus in Korean population. Diabet Med 2005; 22: 569-575.

34 Vozarova de Court B, Hanson RL, Funahashi T et al: Common polymorphisms in the adiponectin gene ACDC are not associated with diabetes in Pima Indians. Diabetes 2005; 54: 284-289.

35 Schaffler A, Herfarth H, Paul G et al: Identification of influencing variables on adiponectin serum levels in diabetes mellitus type 1 and type 2. Exp Clin Endocrinol Diabetes 2004; 112: 383-389.

36 Ohashi K, Ouchi N, Kihara S et al: Adiponectin I164 T mutation is associated with the metabolic syndrome and coronary artery disease. J Am Coll Cardiol 2004; 43: 1195-1200.

37 Esterbauer H, Schneitler C, Oberkofler H et al: A common polymorphism in the promoter of UCP2 is associated with decreased risk of obesity in middle-aged humans. Nat Genet 2001; 28: 178-183.

38 Espeland MA, Hoen H, Byington R, Howard G, Riley WA Furberg CD: Spatial distribution of carotid intimal-medial thickness as measured by B-mode ultrasonography. Stroke 1994; 25: $1812-1819$.

39 Weidinger S, Klopp N, Wagenpfeil S et al: Association of a STAT 6 haplotype with elevated serum IgE levels in a population based cohort of white adults. J Med Genet 2004; 41: 658-663.

40 McCarthy MI: Growing evidence for diabetes susceptibility genes from genome scan data. Curr Diab Rep 2003; 3: 159-167.

$41 \mathrm{Hu} F B$, Doria A, Li T et al: Genetic variation at the adiponectin locus and risk of type 2 diabetes in women. Diabetes 2004; 53: 209-213.

42 Conne B, Stutz A, Vassalli JD: The $3^{\prime}$ untranslated region of messenger RNA: a molecular 'hotspot' for pathology? Nat Med 2000; 6: 637-641. 